\title{
EchoGéo
}

$20 \mid 2012$

Des mobilités aux transports. Regards croisés en Afrique de l'ouest

\section{Afriques noires et Afriques ambiguës.}

Lecture croisée de deux livres sur l'Afrique au sud du Sahara

Henri Nicolaï

\section{OpenEdition}

Journals

Édition électronique

URL : https://journals.openedition.org/echogeo/13104

DOI : 10.4000/echogeo.13104

ISSN : 1963-1197

Éditeur

Pôle de recherche pour l'organisation et la diffusion de l'information géographique (CNRS UMR 8586)

\section{Référence électronique}

Henri Nicolaï, «Afriques noires et Afriques ambiquës. », EchoGéo [En ligne], 20 | 2012, mis en ligne le 13 juillet 2012, consulté le 11 août 2021. URL : http://journals.openedition.org/echogeo/13104 ; DOI : https://doi.org/10.4000/echogeo.13104

Ce document a été généré automatiquement le 11 août 2021.

EchoGéo est mis à disposition selon les termes de la licence Creative Commons Attribution - Pas d'Utilisation Commerciale - Pas de Modification 4.0 International (CC BY-NC-ND) 


\title{
Afriques noires et Afriques ambiguës.
}

\author{
Lecture croisée de deux livres sur l'Afrique au sud du Sahara
}

\section{Henri Nicolaï}

1 Une lecture croisée comme celle qui m'a été demandée pour la nouvelle version de deux livres portant sur l'Afrique au sud du Sahara, est un exercice qui n'est pas sans embûches. Le résultat risque de ne pas satisfaire celui qui l'a entrepris et est menacé d'autre part du reproche de parti pris.

2 Il s'agit de deux livres de même taille, l'un de Roland Pourtier (que nous désignerons par la suite par P), l'autre d'Alain Dubresson, Sophie Moreau, Jean-Pierre Raison et Jean-Fabien Steck (que nous désignerons par l'initiale des auteurs de la première édition, soit D-R), publiés dans des collections analogues par deux maisons d'édition concurrentes et répondant à un objectif identique. Les auteurs, du moins dans la première version, sont tous les trois à peu près de la même génération et n'appartiennent pas à des écoles de pensée vraiment différentes. La première version de l'un (D-R) date de 1998, celle de l'autre (P), de 2001. Les auteurs ont estimé au même moment qu'il fallait revoir ce qu'ils avaient écrit une décennie plus tôt tant l'évolution récente de l'Afrique a été rapide. L'un d'eux $(\mathrm{P})$ a même considéré que la première édition correspondait à la fin d'un cycle et qu'un nouveau avait commencé le 11 septembre 2001, Pour R-D, même constatation: à deux décennies de crises enchevêtrées (RD) a succédé une décennie de croissance économique. Bien sûr les deux ouvrages n'ont pas été totalement refondus. C'est l'introduction et la conclusion surtout qui ont été modifiées avec de nouvelles interrogations sur les perspectives nées des derniers soubresauts. Cependant, dans D-R notamment (mais nous n'avons pas eu la possibilité de relire la première .version du livre $\mathrm{P}$ ), des parts importantes de chapitres ont été récrites. L'ordre a été parfois modifié Les documents annexés aux différents chapitres ont souvent fait place à des textes plus récents ou portant sur des matières nouvelles.

3 Une lecture croisée court le risque d'être décevante dans le cas où, comme dans celuici, les livres ne sont pas antagonistes. Il n'y a pas ici d'affrontement idéologique. Dans la 
perception des problèmes, il y a beaucoup plus de similitudes que de différences, plus de convergences que de divergences, davantage de nuances que d'oppositions. Cela se marque parfois par des réflexions quasi identiques. Par exemple sur le rôle de l'État, la place prise par les ONG (MSF gérant la collecte dans une localité de Madagascar), le mécanisme des REDD. Certains titres de chapitres sont très proches ou pratiquement semblables: la révolution urbaine dans un livre, les transformations urbaines dans l'autre, les paysanneries face au développement d'un côté, les mutations des agricultures de l'autre. Relever les nuances serait un travail de bénédictin à l'utilité contestable.

4 Les deux livres ont cherché à singulariser leurs points de vue dans le titre ou le soustitre. L'un (P) parle directement des Afriques noires et, par l'usage du pluriel, met en évidence la diversité des situations. L'autre (D-R), a choisi une localisation géographique, l'Afrique subsaharienne, expression que, pour notre part, cela dit en passant, nous n'aimons guère. Mais le sous-titre, Une géographie du changement, met en exergue la notion d'un espace en mouvement, en proie à de multiples mutations.

Il n'y a pas là cependant de différenciation rigide. Ainsi le premier livre (P) évoque lui aussi, d'entrée de jeu, des situations de changements, qualifiées plus précisément de crises : marginalisation d'un continent par la mondialisation, crises des États, des paysanneries, des citadins.

6 Dans les deux cas, les ouvrages ont choisi de traiter l'Afrique noire de façon générale (les problèmes démographiques, les problèmes des paysanneries, les problèmes de gestion de l'espace, les problèmes urbains, etc.) et de ne pas envisager systématiquement les compositions régionales si ce n'est de façon assez sommaire. Pour le premier livre, cela peut paraître quelque peu contradictoire avec l'option de diversité affirmée au départ. Certes, dans ses premières pages, il décrit les découpages principaux du continent (y compris les divers essais d'associations officielles d'États, plus ou moins fonctionnelles) mais par la suite, la notion de diversité n'apparaît plus avec la même force, sinon dans des chapitres qui distinguent quelques particularités régionales comme lorsque, par exemple, l'Afrique des greniers (grossièrement celle des savanes) est opposée à celle des paniers (celle de la grande forêt).

7 Le second livre apparaît plus constamment fidèle à l'option qu'il a annoncée puisque ses différents chapitres sont construits sur la notion de changement (changements ou mutations dans les encadrements, l'évolution démographique, les agricultures, les modèles urbains, recomposition des espaces).

8 On trouvera, dans les conclusions respectives, un autre exemple des difficultés que l'on rencontrerait si l'on voulait opposer les deux livres. D-R, qui organise sa démarche autour des changements et des mutations, comporte dans ses dernières lignes, une réflexion que l'on aurait attendue plutôt de $\mathrm{P}$ dont on sait l'importance qu'il attribue aux systèmes politiques, et particulièrement à l'État: L'Afrique subsaharienne a moins besoin de discours apitoyés et d'ingérence humanitaire que de volontarisme public et de projets de sociétés mobilisateurs à toutes les échelles (p.238) et, à la fin du texte de couverture, cette mention l'Afrique subsaharienne est devenue de plus en plus différenciée et plurielle. De son côté, c'est sur le mot "changement », leitmotiv de D-R, que se termine le livre P : C'est à elle [une nouvelle génération] qu'incombe désormais de poursuivre le changement (p. 231).

9 Les deux livres sont d'une étonnante densité. Les auteurs abordent à peu près tous les problèmes et ont le souci de traiter jusqu'à l'actualité la plus récente, par exemple dans le livre $\mathrm{P}$, les effervescences religieuses, les enfants des rues et les enfants soldats, les 
perspectives touristiques d'une Afrique, soleil de l'Europe, les apports de l'Afrique à la vie culturelle mondiale. De ce point de vue, le livre $\mathrm{P}$ apparaît le plus encyclopédique. Il est aussi écrit dans un style différent, un style plus journalistique, sans donner à cette qualification une signification péjorative. Le style de l'autre livre, très homogène malgré le nombre des auteurs, apparait plus complexe.

De façon un peu surprenante, l'ouvrage D-R donne l'impression de prendre à peine en compte les caractères physiques du continent. Il considère en effet que ceux-ci ont été traités dans L'Afrique de Pierre Gourou (Hachette, 1970) et dans Les Afriques au sud du Sahara (tome VI de la Géographie Universelle, Belin-Reclus, 1994) et qu'il n'est pas nécessaire de revenir sur le sujet. C'est à ces deux ouvrages aussi d'ailleurs que les deux livres renvoient le lecteur pour les aspects spécifiquement régionaux. Le livre D-R rappelle simplement que le milieu physique africain a été un champ privilégié d'application des théories déterministes et ne l'évoque qu'à propos des crises environnementales actuelles, dans un chapitre qui ne figurait pas dans la première édition et qui a été écrit par un auteur qui a rejoint l'équipe initiale (L'environnement entre crises et mythes). Il rappelle que les descriptions des relations entre la nature et les sociétés en Afrique ont été dominées depuis près d'un siècle par le paradigme de la dégradation : déforestation, dégradation des sols, désertification et maintenant érosion de la biodiversité végétale et animale (p.174-175) auxquelles viendraient s'ajouter les effets des crises climatiques. Il importe donc de relativiser tout ce qui a été dit sur ces questions auxquelles se rattachent aujourd'hui les projets de développement durable (avec les dérives que peuvent présenter des programmes comme celui de la Réduction des émissions issues de la déforestation et de la dégradation ou REDD, cette alliance de la préservation de la biodiversité et du capitalisme mondial, dont certains fonctionnent déjà en RDC par exemple ou dans les régions sahéliennes).

11 Le livre $\mathrm{P}$ consacre par contre un chapitre entier à la nature (le livre D-R y renvoie d'ailleurs ses propres lecteurs) et aux relations entre milieux et sociétés. Mais il ne le place pas en tête de l'ouvrage comme le faisaient naguère les thèses de géographie régionale. Certes dès le départ, il a insisté sur l'importance de la césure du Sahara. Il effleure ensuite la question de l'effet du milieu naturel à propos de l'histoire démographique, où quelques pages sont coiffées par le titre: démographie, nature et histoire. Celles-ci traitent notamment les relations entre les systèmes pathogènes et le peuplement et, dans un raccourci suggestif, présentent l'insecte et le négrier comme des facteurs fondamentaux de l'histoire africaine. Le chapitre 3 enfin envisage le thème $\mathrm{du}$ milieu naturel de façon systématique. On pourrait dire que par là le livre de $\mathrm{P}$ apparaît plus conforme à une construction académique que celui de D-R.

Ce chapitre sur le milieu physique est aussi l'occasion pour P. de rappeler que l'époque coloniale a vu l'Afrique comme un espace essentiellement sauvage, ce qui lui permettait de minimiser voire de nier le rôle que les hommes avaient pu y jouer auparavant, imposant ainsi l'idée de territoires sans mémoires. Cette négation aurait été d'ailleurs illustrée par la dénomination des territoires. Celle-ci ne fait référence qu'à des faits physiques (fleuves, lacs ou montagnes, parfois ressources naturelles comme l'ivoire ou l'or) et quasi jamais à des faits humains, sinon dans quelques cas comme le Rwanda ou l'Ouganda. L'idée n'est pas inexacte sans doute mais il faut rappeler quand même que le principe avait été retenu par les révolutionnaires pour nommer les départements de la France (en effaçant, il est vrai, par là aussi, les traces de l'Ancien Régime). 

des encadrements, c'est-à-dire, pour être bref, de ces techniques qui, pour Pierre Gourou, règlent les relations des hommes entre eux sur un territoire donné et organisent aussi sa mise en valeur. On s'y attendait dans le livre R-D puisqu'on sait l'importance qu'un des auteurs, Jean-Pierre Raison, attache à la pensée géographique de Pierre Gourou. On s'y attendait moins dans le livre de Roland Pourtier dont les travaux s'en distancient davantage. Mais très logiquement, en abordant l'étude du monde paysan face au développement, celui-ci recourt à la notion d'encadrement en faisant référence au dernier ouvrage écrit par Pierre Gourou (L'Afrique tropicale, nain ou géant agricole?). Il rappelle que, pour Gourou, la rénovation agricole de l'Afrique pluvieuse "dépend avant tout de l'aménagement des techniques d'encadrement" que d'ailleurs « seuls des Africains peuvent...faire » (p. 81). C'est aussi, en parlant du succès des Bamiléké, des Mossi en Côte d'Ivoire, des commerçants soninké, dioula et haoussa, qu'il souligne que chaque cas montrerait que l'efficacité de leurs techniques de production est en partie redevable, pour reprendre le schéma général d'explication de Pierre Gourou, à leurs techniques d'encadrement (p. 134).

Chez R-D, la notion d'encadrement est un élément structurel fondamental. Dès l'introduction, il insiste sur la nécessité qu'ont les sociétés africaines de disposer d'encadrements efficaces pour assurer leur survie et leur modernisation dans le contexte mondial actuel (p. 6). Le chapitre 2 est totalement consacré à cette question sous un titre conforme à la ligne générale de l'ouvrage, Encadrements en mouvement. Ceux-ci englobent la famille, le village, l'ethnie, la région, les réseaux marchands, les hybridations et les ruptures urbaines, où les relations du rural et de l'urbain sont vues comme une géographie du va-et-vient. Ils s'expriment aussi aujourd'hui par de multiples bouillonnements dont le foisonnement religieux illustré par un document annexe en clin d'oeil sur Madame Gbagbo et les évangélistes (la conversion au pentecôtisme de Simone, élue du ciel). Le premier chapitre a envisagé les encadrements par le haut, c'est-à-direles complexes politico-économiques de l'époque moderne (l'État post-colonial et son système d'accumulation rentière extensive, les interventions internationales récentes, la signification de l'informel dans le système actuel). D-R revient sur la notion d'encadrement à la fin de l'ouvrage quand il décrit le dépérissement de l'Etat, évoque l'échec des tentatives d'intégration régionale et développe le thème, familier à un de ses auteurs, des espaces recomposés (espaces transfrontaliers et corridors) qui sont parmi les conséquences de ce dépérissement.

Sans utiliser systématiquement le terme, $P$ décrit le rôle et le poids des encadrements et leur évolution dans un chapitre (chapitre 5) intitulé Le territoire: espace et nation. Fidèle au principe que l'État précède la nation et que le cadre étatique constitue le paramètre premier de la diversité, il étudie le partage colonial de l'Afrique avant de parler des héritages, montre le rôle de l'État dans la construction du territoire, étudie ses relations avec l'ethnie et s'interroge sur le lien entre l'ethnogenèse et la modernité. Mais plusieurs pages sont consacrées aussi aux « encadrements au quotidien »

Le souci général des deux ouvrages de maintenir un optimisme modéré n'empêche pas quelques réflexions assez sombres. A propos par exemple de cette tendance lourde qu'est l'exception démographique africaine, cette forte croissance que même le sida n'a pu ralentir de façon significative, P., notant que le Rwanda atteindra sans doute une densité de population de 850 habitants au $\mathrm{km}^{2}$ en 2050, écrit cette phrase surprenante (p. 53), « la question démographique ne peut pas être éludée....dans des situations de plus en 
plus fréquentes, le choix se pose crûment entre le préservatif et la kalanchnikov ». Après avoir exalté un continent de la jeunesse, le propos est à peine moins noir pour ces vecteurs de la violence que peuvent devenir les jeunes laissés pour compte par la crise économique et qui sont exposés à toutes les manipulations des aventuriers de la politique.

Dès leur première édition, les deux ouvrages ont voulu réagir contre l'afropessimisme. Ils refusent de concevoir l'Afrique comme un continent à la dérive, comme une terre de problèmes apparemment insolubles même à longue échéance. Pour D-R par exemple, les situations de crise ne devraient pas être considérées comme la marque d'un naufrage mais comme une rupture donc d'un changement. Les dernières années donnent une nouvelle image de l'Afrique subsaharienne. Mais se trouve-t-on vraiment devant une bifurcation qui l'engagerait dans la voie d'une réelle modernisation? Si les discours actuels valorisent l'idée de nouvelle frontière, l'irruption de la chine et d'autres pays émergents va-t-elle changer les structures économiques ou ne va-t-elle pas enfermer à nouveau l'Afrique dans l'impasse qu'a été la fonction de fournisseur de matières premières et d'hommes qu'on lui a attribuée depuis plusieurs siècles? Les handicaps de l'Afrique au cours des trois dernières décennies (faiblesse de la productivité, défaillance des infrastructures, aggravations des conditions sanitaires) étaient sans doute moins importants que la carence des encadrements, et d'abord des encadrements étatiques.

Pour marquer l'incertitude de l'avenir, la conclusion de D-R reprend le titre de l'ouvrage qu'avait écrit Georges Balandier, dans la collection Terre Humaine, en 1957. En le mettant au pluriel, Afriques ambiguës, il insiste lui aussi sur la diversité des évolutions.

Cette lecture croisée, s'il faut vraiment l'achever par une conclusion, ne peut aboutir à l'artifice qui consisterait à dire que les deux livres sont complémentaires. En fait il s'agit de visions parallèles qui, dans la richesse de leurs développements, doivent nourrir toutes deux les réflexions des géographes.

\section{BIBLIOGRAPHIE}

Pourtier R., 2010. Afrique noires, $2^{e}$ édition revue et augmentée. Carré Géographie, Hachette Supérieur, Paris, $282 \mathrm{p}$.

Dubresson A., Moreau S., Raison J-P., Steck J-F., 2011. L'Afrique subsaharienne, une géographie du changement, $3^{e}$ édition entièrement revue et augmentée. Collection U, Armand Colin, Paris, 269 p. 